\title{
Rheumatic Fever Licks at the Joints, but Bites at the Heart
}

\author{
Konstantinos Dean Boudoulas ${ }^{a}$ Antonios Pitsis $^{b}$ Harisios Boudoulas ${ }^{\mathrm{a}, \mathrm{c}}$ \\ a Department of Medicine, Division of Cardiovascular Medicine, The Ohio State University, Columbus, OH, USA; \\ ${ }^{b}$ Department of Cardiothoracic Surgery, St. Luke's Hospital, Thessaloniki, Greece; 'Biomedical Research Foundation, \\ Academy of Athens, Athens, Greece
}

Rheumatic fever is the result of an abnormal autoimmune response to group A streptococcal pharyngeal infection in a susceptible host. However, in approximately one-third of cases a history of pharyngeal infection is not elucidated prior to the development of rheumatic fever. In certain individuals, which is not uncommon, streptococcal pharyngeal infection does not result in rheumatic fever. As a general rule, rheumatic fever affects young individuals (6-15 years old), but can occur in adulthood. The clinical manifestations of rheumatic fever include migratory arthritis, fever, erythema marginatum (nonpruritic rash with sharp erythematous borders), subcutaneous nodules, chorea, and carditis [1-3].

During the acute phase of the disease, typically the left sided cardiac valves appear edematous and inflamed. Tiny verrucae (1-2 $\mathrm{mm}$ in diameter), consisting of platelets and fibrin, are seen along the lines of valve closure. These findings can be detected with current imaging techniques, especially echocardiography. Inflammation involving the connective tissue of the valves can also be seen in the mitral annulus and the chordae tendineae. Mitral regurgitation is the most common clinical presentation of acute valvulitis, ranging from mild to severe [1].

In this issue of Cardiology, Narang et al. [4] report the clinical presentation of 126 children with acute rheumat- ic fever who were treated in a tertiary medical center $(60$ patients had a previous history of rheumatic fever). Joint symptoms were present in $29 \%$ and fever in only $25 \%$ of the patients; $2.4 \%$ of the patients had subcutaneous nodules and there were no patients with erythema marginatum or chorea. Tachycardia and heart failure were present in 53 and $21 \%$ of the patients, respectively. Echocardiogram demonstrated mitral leaflet thickening in $77 \%$, small nodules on the tip of the mitral leaflets in $43 \%$, thickening of the aortic valve in $27 \%$, and nodules in the aortic cusps in $8 \%$ of the patients. Mitral regurgitation ranging from mild to severe was present in $95 \%$, mitral stenosis in $16 \%$, aortic regurgitation in $44 \%$, and tricuspid regurgitation in $56 \%$ of the patients. Of interest, mitral valve prolapse (MVP) was seen in $29 \%$ and flail leaflet was seen in $5 \%$ of the patients. The most recent modified Jones criteria (2015) for the diagnosis of acute rheumatic fever were present in only $32 \%$ of the patients [5].

\section{Natural History of Rheumatic Valvulitis}

After a long latent period, varying from 10 to 40 years, symptoms related to the underlying valvular disease occurs. It should be noted that approximately $50 \%$ of pa-

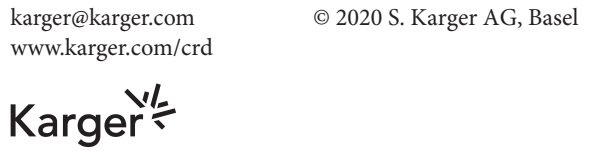

Harisios Boudoulas 


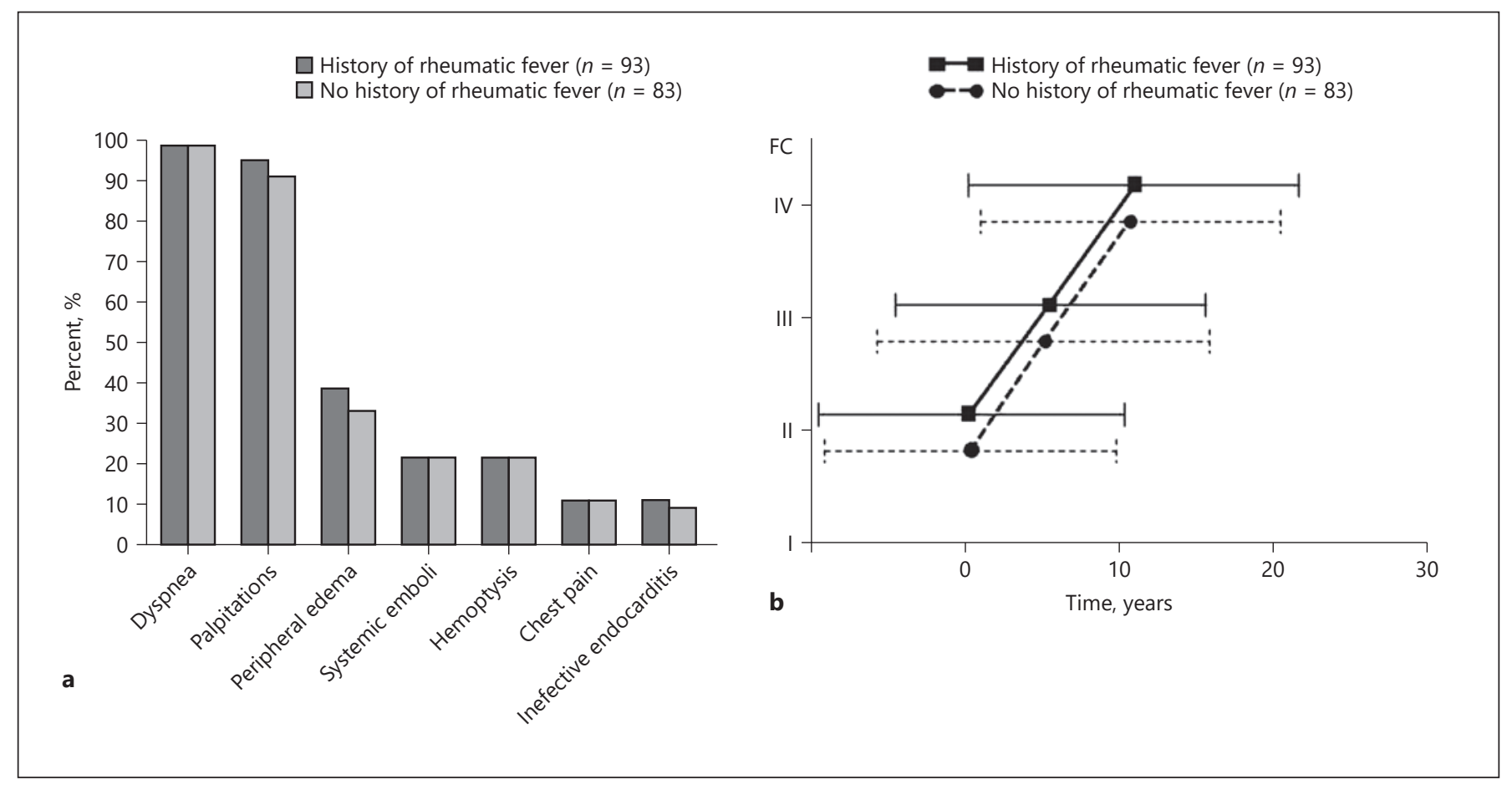

Fig. 1. a Frequency of symptoms/events in patients with mitral stenosis with and without a history of rheumatic fever. Note the similarity between the two groups. b Time intervals from the initiation of symptoms to severe symptoms in patients with mitral stenosis are shown (from Boudoulas and Grvanis [1] and Boudoulas et al. [6]). FC, functional class.

tients with rheumatic valve disease have no history of rheumatic fever; interestingly, after the beginning of symptoms the "natural history" of rheumatic valve disease is the same in patients with no history of rheumatic fever as compared to those with a history of rheumatic fever. In a previous study, 176 patients with mitral stenosis hospitalized in the A' Medical Department of the Aristotle University of Thessaloniki, AHEPA Hospital, Greece, from 1962 to 1972 were analyzed. The average age of symptom onset in patients with a history of rheumatic fever $(n=93)$ was similar to those without a history of rheumatic fever $(n=83): 36.5 \pm 14$ versus $36.7 \pm 13$ years, respectively; however, there was a wide distribution in age at symptom onset in both groups. The frequency of major symptoms was also similar in both groups (Fig. 1a). The time interval from the initiation of symptoms (functional class II) to severe symptoms (functional class IV) was approximately 10 years and was similar in both groups (Fig. 1b). These patients represent the natural history of mitral stenosis in an era where mitral valve surgery was not widely available in Greece and in which valvuloplasty was yet to be introduced into clinical practice; patients were treated only with digitalis and diuretics. The onset of symptoms and the natural course of the disease is similar with other valvular heart disease of rheumatic etiology regardless of a history of rheumatic fever. Mitral stenosis is presented here as it is considered entirely due to rheumatic etiology, if congenital causes are excluded $[1,6]$.

\section{The Fall of Rheumatic Fever}

The incidence of rheumatic fever and rheumatic valve disease has declined dramatically in the USA and other economically developed countries over the last 7-8 decades. Rheumatic fever, however, still remains a major cause of morbidity and mortality in low-income countries and persists in immigrants in economically developed countries. Factors responsible for this decline include socioeconomic improvements in many parts of the world, improvement in medical care, and use of antibiotics. The decline in the incidence of rheumatic fever, however, was noted well before the use of antibiotics [7, 8] (Fig. 2). Changes in the virulence of the microorganisms involved, host susceptibility, or both may account for this phenomenon. Evidence suggests that the incidence of streptococ- 
Fig. 2. Unadjusted rates of death due to rheumatic fever in the USA, 1910-1977 (from Gordis [7]).

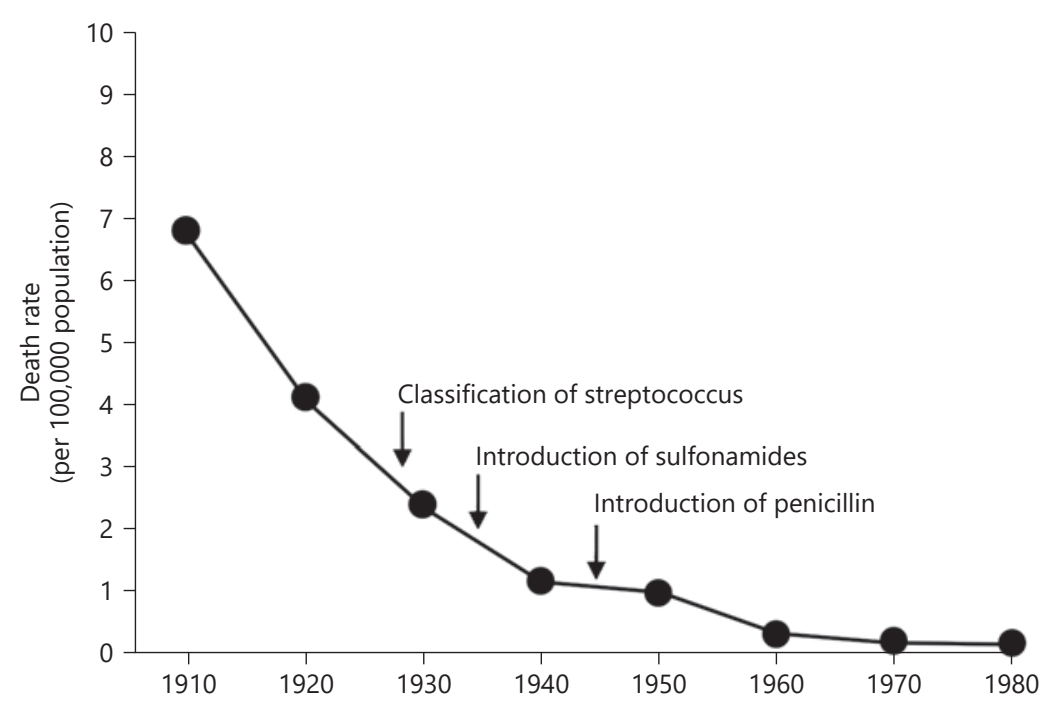

acute rheumatic fever [10]. Of interest, TGF pathways are also related to the pathophysiologic mechanisms of floppy mitral valve (FMV)/MVP [11].

In a study by our group, characteristics of mitral valves in 17 normal (autopsy), 27 mitral stenosis (surgery), and $13 \mathrm{FMV} / \mathrm{MVP}$ (surgery) individuals were evaluated. Values that were 2 standard deviations above the normal mean of mitral annular circumference, mitral annular area, and mitral leaflet area were considered abnormal. All measurements were abnormally large in FMV/MVP patients, which is mostly considered a heritable disease. In mitral stenosis, there was also an increase in mitral annular circumference, mitral annular area, and mitral leaflet area by 37,48 , and $52 \%$ of the cases, respectively [12]. The data suggest that certain patients with mitral stenosis have dimensional characteristics that resemble FMV. Fibrosis, which is the dominant pathologic change in chronic rheumatic mitral stenosis, would not be expected to result in an increase in valvular dimensions. Thus, rheumatic mitral stenosis may more often occur in patients with underlying FMV/MVP.

\section{Concluding Remarks}

The only significant sequela of rheumatic fever is rheumatic valve disease, while arthritis, erythema, and subcutaneous lesions are transient events. Thus, cardiac in- 
volvement should be the main (if not the only) focus in patients with rheumatic fever and diagnostic criteria should be based mostly on echocardiography. However, since approximately half of the patients with rheumatic valve disease have no history of rheumatic fever, no matter what diagnostic criteria are used, the diagnosis of the acute episode could be missed in a large proportion of patients. It follows that attempts should be made to identify high-risk individuals prone to disease and to provide preventive measures in these highly susceptible individuals $[2,7,9,10]$. Genome-wide association studies may help to achieve this goal. Prophylactic measures in this high-risk group, including use of antibiotics and vaccination (when a vaccine is available), will result in a decrease in the incidence and may result in the eradication of rheumatic fever worldwide in the years to come.

\section{Disclosure Statement}

The authors declare that they have no conflicts of interest to disclose.

\section{References}

1 Boudoulas H, Grvanis MB. Valvular heart disease. In: Gravanis MB, editor. Cardiovascular disorders, pathogenesis and pathophysiology. St. Lousi: Mosby-Year Book; 1993. pp. 64-117.

2 Bryant PA, Robins-Browne R, Carapetis JR, Curtis N. Some of the people, some of the time: susceptibility to acute rheumatic fever. Circulation. 2009 Feb;119(5):742-53.

3 Watkins DA, Johnson CO, Colquhoun SM, Karthikeyan G, Beaton A, Bukhman G, et al. Global, regional and national burden of rheumatic heart disease, 1990-2015. N Engl J Med. 2017 Aug;377(8):713-22.

4 Narang R, Saxena A, Ramkrishnan S, et al. Characteristics of children with acute rheumatic carditis from a high incidence region. Importance of unexplained worsening of functional class. Cardiology. doi: 10.1159/000508035.
5 Gewitz MH, Baltimore RS, Tani LY, Sable CA, Shulman ST, Carapetis J, et al.; American Heart Association Committee on Rheumatic Fever, Endocarditis, and Kawasaki Disease of the Council on Cardiovascular Disease in the Young. Revision of the Jones Criteria for the diagnosis of acute rheumatic fever in the era of Doppler echocardiography: a scientific statement from the American Heart Association. Circulation. 2015 May;131(20):1806-18.

6 Boudoulas H, Kontopoulos A, Parharidis G, Metaxas $\mathrm{P}$, Vltis J. The natural history of valvular heart disease. Comparison of patients with a history of rheumatic fever to those without history of rheumatic fever [Greek Medicine]. Helliniki Iatriki. 1974;43:107-15.

7 Gordis L. The virtual disappearance of rheumatic fever in the United States: lessons in the rise and fall of disease. T. Duckett Jones memorial lecture. Circulation. 1985 Dec;72(6): 1155-62.

8 Boudoulas KD, Borer JS, Boudoulas H. Etiology of valvular heart disease in the 21st century. Cardiology. 2013;126(3):139-52.
9 Engel ME, Stander R, Vogel J, Adeyemo AA, Mayosi BM. Genetic susceptibility to acute rheumatic fever: a systematic review and meta-analysis of twin studies. PLoS One. 2011; 6(9):e25326.

10 Muhamed B, Shaboodien G, Engel ME. Genetic variants in rheumatic fever and rheumatic heart disease. Am J Med Genet C Semin Med Genet. 2020 Mar;184(1):159-77.

11 Boudoulas KD, Pitsis AA, Mazzaferri EL, Gumina RJ, Triposkiadis F, Boudoulas H. Floppy mitral valve/mitral valve prolapse: a complex entity with multiple genotypes and phenotypes. Prog Cardiovasc Dis. 2020. doi: 10.1016/j.pcad.2020.03.004

12 Baker PB, Boudoulas H, Kilman J, Wooley CF. Evidence that mitral stenosis may occur on myxomatous mitral valves. Neinvaz Kardiol. 1991;Suppl:A1. 